\title{
Unsteady Pressure Field Investigation of an Axial Fan-Inlet and Outlet Unsteady Pressure Field Measurement
}

\author{
C. Xu, R. S. Amano, and R. Perez \\ Department of Mechanical Engineering, University of Wisconsin-Milwaukee, Milwaukee, \\ Wisconsin, USA
}

The unsteady pressure characteristics at inlet and outlet of an axial fan were measured in this study. A $1.829 \mathrm{~m}(6 \mathrm{ft})$ diameter axial fan was operated at $1770 \mathrm{rpm}$ in a laboratory. The unsteady pressure field was obtained at three axial positions each with seven radial locations. The results showed that there was a relatively long response time for pressure drop both in the inlet and outlet during fan start-up. The measurements also showed that, due to the vortex shedding from the trailing edge of each fan blade, the fan outlet pressure oscillation frequency was related to the fan operating frequency. A theoretical analysis was also conducted in order to understand the measurements. The unsteady pressure measurements helped improve the fan performance and contributed to the understanding of the vibrational behavior of the fan unit. The complete set of the measurements obtained can be used as a database for computational fluid dynamics (CFD) codes validation and modeling.

Keywords Unsteady aerodynamic; Pressure measurement; Axial fan

Axial fans are used in many areas in industry such as mine ventilation, air-conditioning systems, heat exchangers, etc. They are used to discharge air in the area to be ventilated or force air to move into heat exchangers. A fan exchanges momentum with the surrounding secondary air in exactly the same manner as an axial pump or an axial compressor, but a fan can deliver air volume much greater than its own inlet volume. Most industries still use steady two-dimensional wind-tunnel data to estimate aerodynamic loads and to obtain guidelines for fan

Received 25 August 2000; accepted 2 August 2001.

Address correspondence to Ryoichi S. Amano, Mechanical Engineering Department, University of Wisconsin-Milwaukee, EMS Building, P. O. Box 784, Milwaukee, WI 53201.

design and vibration consideration. The two-dimensional methods usually under-predict the actual loading and over-predict the performance. Although fan industries have been developing large flow-rate fans for many years, they still face many difficulties constructing efficient and stable fan units. To solve these problems, an extensive study of fan aerodynamics has been conducted. Because the fan efficiency and corresponding loading is caused by aerodynamic behavior which relates to the fan, the underlying fluid dynamics must be well understood to accurately determine structures. Fan aerodynamics has been extensively investigated for many years. Abramovich (1963) studied the air distribution in a dead-end channel by using an axial fan. The study showed that in a duct system, very high velocities resulted at discharge section but relatively low velocities occurred throughout the body of the flow path. Recently, Mutama and Hall (1996) studied the pressure distributions of a jet fan traveling from one wall along the axis of a tunnel in order to optimize the location of the jet fan. However, few articles report on the dynamic behavior of an axial fan.

The ability to make an unsteady measurement in a complicated geometry greatly enhances the investigative efforts of researchers seeking to understand the behavior of aerodynamics. There have been a number of experimental articles on axial turbomachines (Gallus, Lambertz, and Wallmann, 1980; Dring et al., 1982; Capace and Fleeter, 1989). Most of the studies dealt with the rotor-stator interaction through the velocity field and wake characteristics when an axial turbomachine rotated at a constant speed. More recently, Huyer, Simms, and Robinson (1996) studied the unsteady aerodynamics of a wind turbine associated with a change in yaw angle through steady pressure measurements. In addition, an analysis was performed regarding the unsteady aerodynamics. The studies associated with high-density unsteady flow characteristics are difficult to find in the open literature. In recent years there have been substantial developments in the variety and quality of support facilities available for research in experimental aerodynamics, especially the advances in pressure transducer manufacturing 

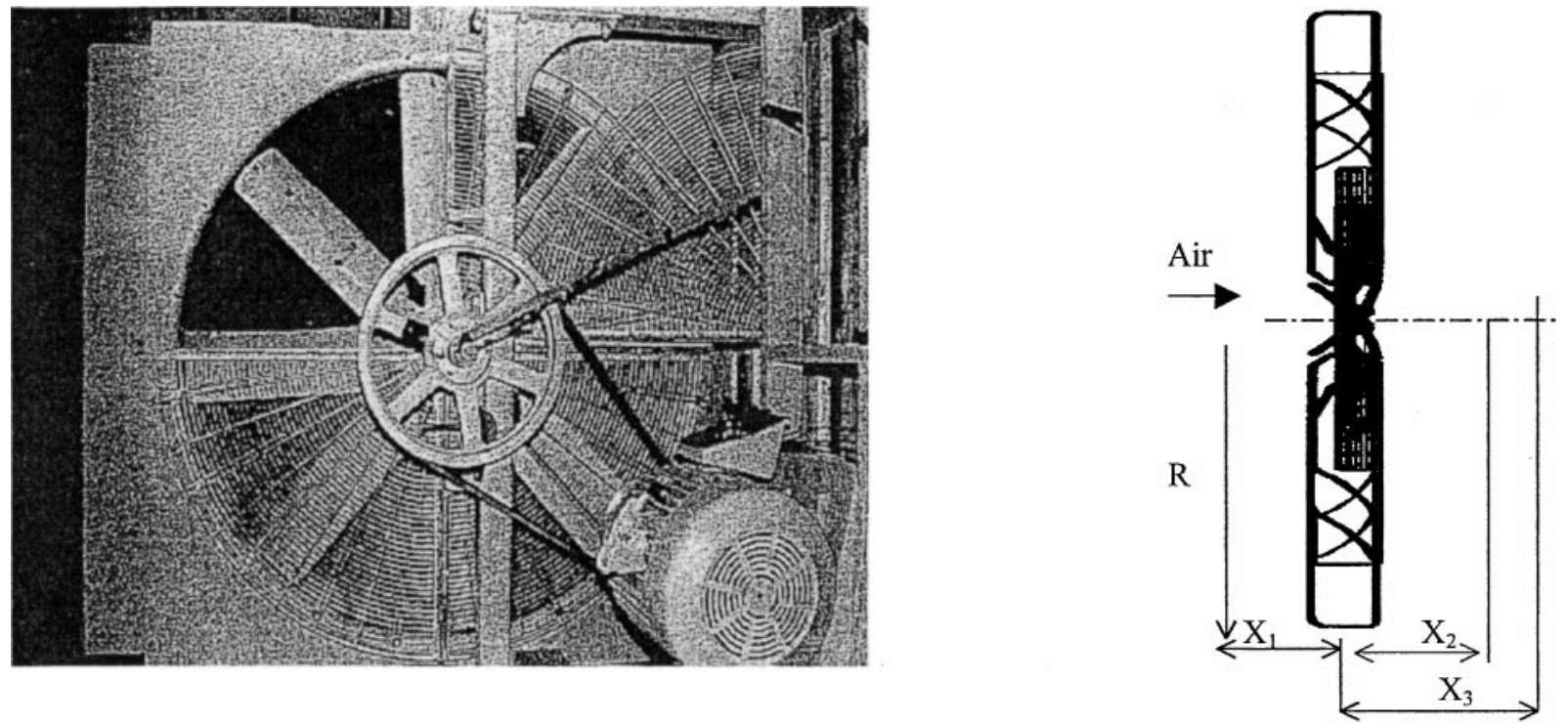

FIGURE 1

Test fan and pressure transducer positions.

technology and the capabilities of PC-based data acquisition systems that can significantly reduce the cost of the unsteady pressure field measurement. Kaupert and Staubli (1999a, 1999b) extensively investigated the unsteady pressure field in a centrifugal pump impeller. Their measurements comprised two parts; one is the influence of varying the volume flux on the pressure field due to the influence of the pump volute, and the other is an unsteady field study of the transient hysteresis in the characteristics. Their studies used piezo-resistive pressure transducers to obtain the unsteady pressure distributions. However, unsteady measurements related to the axial fan are still limited. The present work is aimed at the unsteady aerodynamic study of an axial fan by providing some fundamental data on the subject. The main objectives of this study are to investigate the unsteady pressure fields of the inlet and outlet of the fan during the fan transition from the start-up to reaching its operating speed.

In this study, the unsteady pressure in the inlet and outlet of the fan in the stationary frame, influenced by rotational fan blades are studied experimentally. Considerable attention has also been given to the role of high-quality experimental measurement to validate CFD codes, especially the unsteady flow simulation codes. Discussions of the unsteady aerodynamic flow produced by an axial fan provide increased understanding of the unsteady flow fields generated by other rotational machines as submarine propellers, helicopter rotors, and turbomachinery.

\section{TEST FACILITY AND INSTRUMENTATION}

An eight-blade industrial axial fan designed for ventilation and heat exchangers was used as the test bed in this study. The outer diameter of the blades is $d_{2}=1.829 \mathrm{~m}(6 \mathrm{ft})$ and the inner diameter of the blades is $d_{1}=0.305 \mathrm{~m}(1 \mathrm{ft})$. The axial fan is installed at the axial center of a streamline-shaped casing and the casing length is $0.37 \mathrm{~m}$ with a diameter of $1.84 \mathrm{~m}$ at inlet. A staggered-angle of the fan-blade from the rotation plane is $30^{\circ}$. The fan blade was designed with a constant chord length along the radial direction. The chord length of the fan is $C=0.246 \mathrm{~m}$. Details of the fan are shown in Figure 1. The experiments were performed at a steady rotational speed of $1770 \mathrm{rpm}$ (tip velocity of the blade is about $u_{t}=169.5 \mathrm{~m} / \mathrm{s}$ ). A Dynamometer was used to verify the rotational speed. The laboratory temperature is about $20^{\circ} \mathrm{C}$ and the Reynolds number based on the blade diameter was of the order of $10^{6}$ at $\omega=1770 \mathrm{rpm}$. The dynamic pressure measurement systems with measurement uncertainty of pressure and time delay are

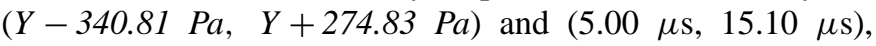
respectively. As reported by Xu and Amano (1999), these uncertainties are less than $10 \%$ of the minimum dynamic pressure. The eigenfrequency of the pressure transducer in air was near $500 \mathrm{kHz}$. This was well above the frequencies of interest $(2 \mathrm{kHz})$. The small cavity in front of the transducer, which was designed to measure the total pressure, revealed no resonance problems near the frequencies of interest.

The low pass (LP) filter frequency setting is one of the important factors of dynamic measurement system. The common method is to let the LP filter frequency equal to the Nyquist frequency in order to minimize the digitization errors. The Nyquist frequency is usually set at half of the sampling frequency. In this study, dynamic pressure data $P$ was collected at a sample rate of $1 \mathrm{kHz}$ and alias filtered at about $300 \mathrm{~Hz}$. Atmospheric pressure $P_{\infty}$ was subtracted due to the design of the pressure transducer. To quantify the effect of the measured drift and repeatability on the bias error, three sets of the test data for each position 
were collected and compared. Various procedures were used to ensure that the test system maintained the required accuracy levels. In some of the instrumentation, a two-step calibration procedure was used. The first part relied on the factory-supplied transducer calibration report which is traceable to NIST (USA) and the second part is an electronic calibration, where precision reference voltages are introduced in place of the transducer signal, and a system electronics path is calibrated (Xu and Amano, 1999). The signal analysis when the fan is in a steady operation was performed for a sampling set of $2^{15}$ points with sample frequency about $1 \mathrm{kHz}$, which is slightly more than 966 rotations of the fan. For the data analysis of the fan starting, a sample set of $2^{18}$ points, with a sample frequency about $0.5 \mathrm{kHz}$ was used. Phase averaging of the unsteady measurement is a common practice in the unsteady measurement (Minniti, Sullivan, and Mueller, 1998) to eliminate a part of the signal often linked to the stochastic effects and to reduce the size of the data sample which helps in interpreting the rotational harmonics of the signal. The signals were phase averaged in this study. A smooth technique was used to represent the unsteady aerodynamic of the fan during start-up and until it reached its operating speed.

The pressure transducer was mounted on a guide ruler which is fixed on a stand. Three axial positions were measured: one inlet position at distance of $X_{1}=0.273 \mathrm{~m}$ upstream from the fan and two axial positions in the outlet at a distance of $X_{2}=0.074 \mathrm{~m}$ downstream, which is very near the fan blade, and at $X_{3}=$ $0.186 \mathrm{~m}$ downstream, which coincides with the outlet plane of the casing, respectively. There were seven radial pressure transducer positions in each test station, i.e., $R_{1}=0.25, R_{2}=0.33$, $R_{3}=0.50, R_{4}=0.67, R_{5}=0.83, R_{6}=0.92, R_{7}=1.00$.

The lowest pressure $p_{m}=8481 \mathrm{~Pa}(1.23 \mathrm{psi})$ in the test station $X_{3}=0.186 \mathrm{~m}, R_{4}=0.67$ was used as a reference pressure for the pressure coefficient. This reference location is near the geometrical center of the blade. The measurement data are all presented in non-dimensional forms with a reference pressure to form the pressure coefficient given by:

$$
C_{p}=\frac{p}{p_{m}}
$$

More than three runs were performed in each test point without any noticeable change in the unsteady pressure responses. Therefore, it is concluded that the present measurement system is robust.

\section{THEORETICAL ANALYSIS}

The unsteady flow phenomenon caused by a fan is a very complex issue, and very few theories are available to correctly explain it. To enhance our knowledge of the unsteadiness in the rotational machine and to better understand the experimental results, some of the theories associated with the fan aerodynamics are discussed here.

According to the study by Xu and Yeung (1998), the pressure downstream of a rotation machine and a airfoil moving spoiler can be simulated by using the vortex method. The pressure oscillations downstream of the fan are significantly affected by many factors, including the environmental conditions during the testing. For the purpose of understanding the basic mechanism of the downstream pressure oscillation of the fan, two-dimensional, inviscid, incompressible cavitation-free flow was employed. At this condition, the flow can be described as a two-dimensional potential flow. Consider the velocity induced by the vortices at the point $(r, \theta)$ in the stationary coordinate system. If the fan blade bounded vortex strength is $\gamma$, according to the basic formulation discussed (Xu and Yeung, 1998), the velocity induced by $\gamma$ downstream of the fan can be expressed in Fourier series as:

$$
\begin{aligned}
& V_{r b}=-\frac{Z}{2 \pi r} \int_{s 1}^{s 2}\left\{\sum_{n=1}^{\infty}\left[r_{b} / r\right]^{n Z} \sin \left[n Z\left(\theta-\theta_{b}-\omega t\right]\right\} \gamma d s\right. \\
& V_{\theta b}=-\frac{Z}{2 \pi r} \int_{s 1}^{s 2}\left\{1+\sum_{n=1}^{\infty}\left[r_{b} / r\right]^{n Z} \cos \left[n Z\left(\theta-\theta_{b}-\omega t\right]\right\} \gamma d s\right.
\end{aligned}
$$

The vortex shedding rate can be calculated by using Helmholz theorem (Xu and Yeung, 1998), that is the total circulation change around the fan blade which is equal to the total shed vortices form the trailing edge within unit time. Then the velocity induced by the vortices shed from the fan blade trailing edge $\gamma_{w}$ can be expressed as:

$$
\begin{aligned}
V_{r w}= & -\sum_{q=1}^{Z} \int_{s 2}^{\infty} \frac{1}{2 \pi r} \frac{\left(r_{q} / r\right) \sin \left(\theta-\theta_{q}\right)}{1+\left(r_{q} / r\right)^{2}-2\left(r_{q} / r\right) \cos \left(\theta-\theta_{q}\right)} \\
& \times \gamma_{w q} d s \\
V_{\theta w}= & -\sum_{q=1}^{Z} \int_{s 2}^{\infty} \frac{1}{2 \pi r} \frac{\left(r_{q} / r\right) \cos \left(\theta-\theta_{q}\right)}{1+\left(r_{q} / r\right)^{2}-2\left(r_{q} / r\right) \cos \left(\theta-\theta_{q}\right)} \\
& \times \gamma_{w q} d s
\end{aligned}
$$

The velocity induced by blade bounded vortices and shed vortices can be written as:

$$
\begin{aligned}
& V_{r}=V_{r b}+V_{r w} \\
& V_{\theta}=V_{\theta b}+V_{\theta w}
\end{aligned}
$$

The total velocity can then be expressed as:

$$
V=V_{r}+V_{\theta}
$$

From Equations (6) and (7), the velocity field downstream of the fan is found to be induced by the blade bounded vortices which are due to the rotation of the fan and the vortex shedding from the trailing edge of the blade. The unsteady pressure can be calculated using the following unsteady Bernoulli's equation:

$$
p / \rho+V^{2} / 2+\partial \phi / \partial t-\left(p_{\infty} / \rho+V_{\infty}^{2} / 2+\partial \phi / \partial t\right)=g H
$$




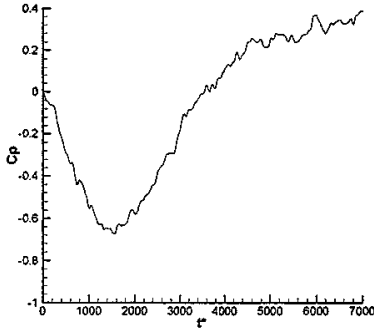

(a) $\mathrm{R}_{2}=0.25$

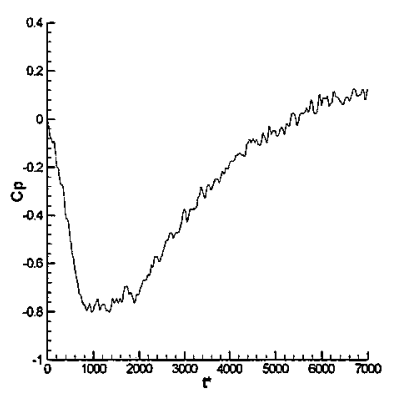

(d) $\mathrm{R}_{4}=0.67$

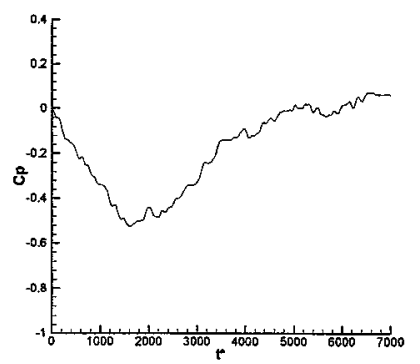

(b). $\mathrm{R}_{2}=0.33$

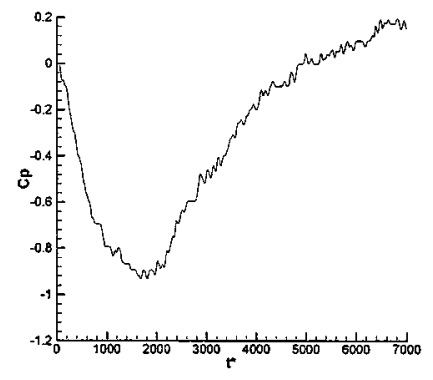

(e) $\mathrm{R}_{5}=0.83$

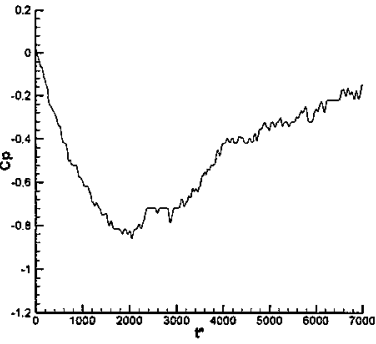

(c) $\mathrm{R}_{3}=0.50$

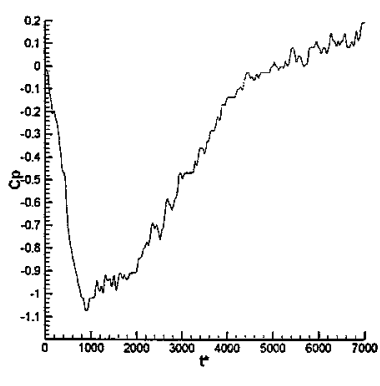

(f) $\mathrm{R}_{6}=0.92$

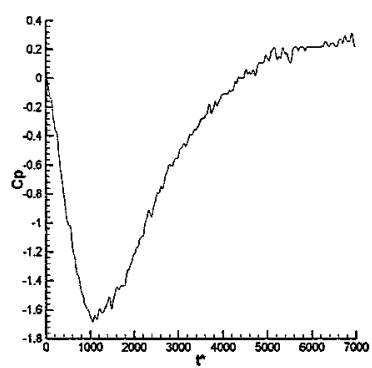

(g) $R_{7}=1.00$

FIGURE 2

Unsteady pressure at fan inlet at $X_{1}=0.273$.

where:

$$
\partial \phi / \partial t=Z \omega \gamma d s / 2 \pi \sum_{q=1}^{\infty}\left(r / r_{b}\right)^{-q Z} \cos \left[q Z\left(\theta-\theta_{b}+\omega t\right)\right]
$$

From Equations (2) to (9), we can obtain the unsteady pressure formulation. It is shown that from Equations (2) to (10), the pressure field was primarily a sinusoidal oscillation. The pressure oscillation frequency depends on the fan rotational speed. The detailed analysis of the unsteady pressure can be found in Xu and Yeung (1998). In this work, the above formulation will be used to explain the measurement results in the following sections.

\section{RESULTS AND DISCUSSION}

\section{Unsteady Pressure at the Fan Startup Stage}

During the fan start process, the fan starts from rest and accelerates to the designed rotational speed. Due to the strong aerodynamic interactions between the air and the blades, the flow characteristics are very complicated and highly unsteady. The unsteady sources at the fan start stage are determined by both the vortices shedding from the fan blades and the change in the rotational speed. It is necessary to understand the fan pressure characteristics during the fan start-up. The Theoretical Analysis section showed that the unsteady pressure characteristics of the fan were found to be affected significantly by the axial position and the rotational speed. A two-dimensional analysis can provide some information about the unsteady pressure characteristics.

Figure 2 shows the unsteady pressure distributions $C_{p}$, with the non-dimensional time, $t^{*}$, at the inlet of the fan. It is shown that it takes around $t^{*}=7000$ to reach the aerodynamic steady state which is longer than the mechanical steady state of the fan (the fan takes about $t^{*}=5000$ to reach a steady speed of $1770 \mathrm{rpm})$. These results show that there are similar trends in all radial directions. The pressure drops from the ambient pressure shortly after start up and monotonically climbs up to a steady level. When the fan is started, the pressure energy of the air is reduced and it builds up the momentum energy that allows the air movement into the fan, which causes very strong unsteady turbulent circulation vortices. This strong unsteady turbulent flow causes a large pressure loss. The unsteady pressure decreases to the lowest level, then the pressure recovers as time increases. However, it is noteworthy to point out that the time of the minimum pressure occurrence varies in different axial locations. It seems that there exists a lowest minimum pressure at the tip $\left(R_{7}=1\right)$. This is because as $R$ increases, the circular velocity of the fan blade increase. The velocity energy portion also increases as the radius increases, which is caused by the pressure drop. It is interesting to point out that the minimum pressure at $R_{1}=0.25$ is lower than at $R_{2}=0.33$ as show in Figures 2(a) and $2(b)$. This is because, in the current fan design, the motor belt casing (see Figure 1) is located close to the position of $R_{1}=0.25$. Therefore, this casing blocks the air and hence forms a separation zone, which causes the lowest pressure at that location. A general trend shown in Figure 2 is that a long-term 


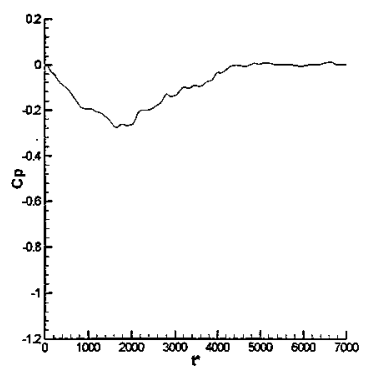

(a) $\mathrm{R}_{1}=0.25$

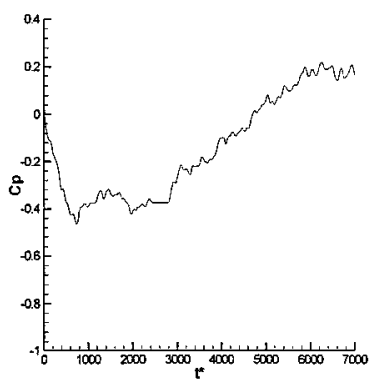

(d). $\mathrm{R}_{4}=0.67$

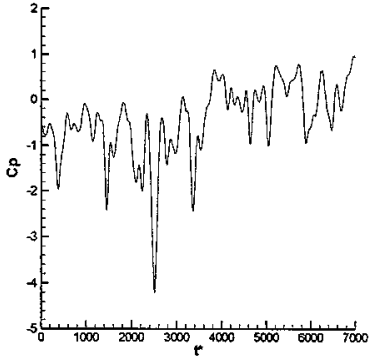

(b) $\mathrm{R}_{2}=0.33$

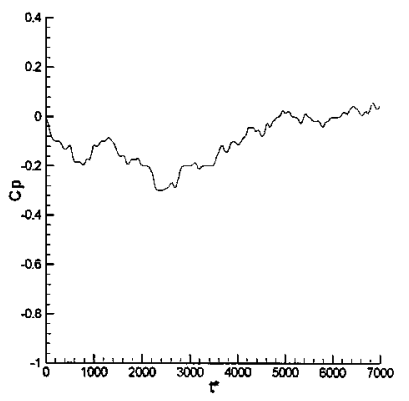

(e) $\mathrm{R}_{5}=0.83$

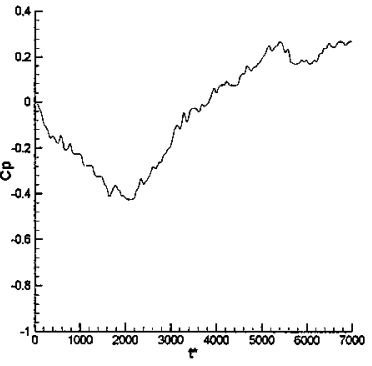

(c) $\mathrm{R}_{3}=0.50$

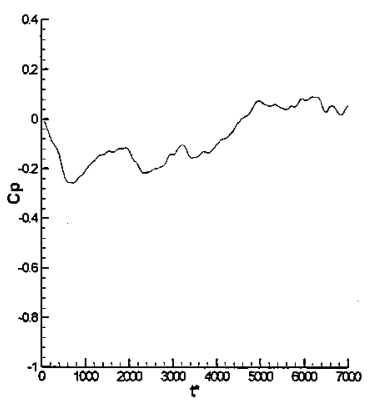

(f). $\mathrm{R}_{6}=0.92$

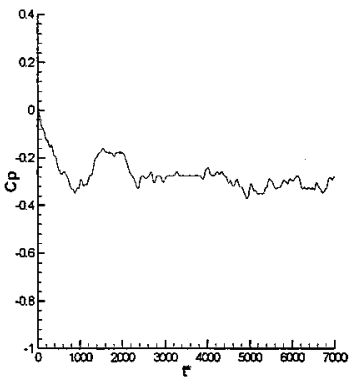

(g). $\mathrm{R}_{7}=1.00$

FIGURE 3

Unsteady pressure during the fan start at $X_{2}=0.074$.

steady pressure is lowest at the middle of the fan $\left(R_{3}=0.5\right)$ and it becomes high near the hub and tip locations of the fan. Shown also in the same figure is a space near the hub due to the connection between the blades and the hub. This space leads to high-energy air at the outlet that tends to flow back to the inlet. This phenomenon certainly causes energy losses and thereby causes the inlet static pressure to increase near the range of the hub. For the same reason, near the tip range of the fan due to the tip clearance, the steady static pressure increases. Finally, it is shown that the time to reach the minimum pressure varies along the radial direction. It seems that near the tip and the hub, the pressure drops faster than near the middle part of the blades.

Figure 3 shows $C_{p}$ changes with $t^{*}$ at $X_{1}=0.074$ downstream from the fan rotational plane. The general trend of $C_{p}$ changes with $t^{*}$ is similar to those observed in the inlet plane. However, the minimum pressure levels at this axial position are much higher than those in the inlet plane. Unlike with the inlet plane a sharp pressure drop occurs only in the middle of the blade radius, i.e. $R=0.50$ and 0.67 . In addition, the change in pressure with time is quite moderate near the hub and tip ranges. The pressure variation near the hub can be explained due to the very low air speed in that range. Since the tangential velocity is very small when near the hub, the rotational blades do not provide very strong influence on the flow field which is consistent with the theoretical analysis described above. The theory indicates that velocities of the air in the outlet of the fan are caused by both rotational speed and the radial direction vortex shedding. From the two-dimensional theory discussed in the theoretical analysis section, it is difficult to explain the variation of the pressure near the tip. According to the two-dimensional theory, the variation near the tip range should be due to the induced airflow. However, in the tip range, the three-dimensional effect plays an important role. The vortex shedding is formed around the tip and the motion of the air strongly interacts with the wall causing strong secondary flows. These interactions can induce large pressure loss. Therefore, the unsteady pressure decreases as the flow developed (i.e. as time progresses). It is shown that less pressure fluctuations occur when the time increases at the radial locations near the casing and hub as depicted in Figure 3. However, the pressure fluctuations are enhanced near the middle of the radial locations of the blade. It is noticed that the pressure oscillates significantly at $R_{2}=0.33$ as shown in Figure $3(b)$, mainly because of the location of this testing point near the trailing edge of the blades. According to the theoretical analysis section, when the locations are near the trailing edge of the blades, the shed vortices strongly influence the flow field and the flow characteristics are dominated by the vortex shedding. That is, the pressure change is mainly determined by the vortex shedding. This causes the pressure to oscillate strongly with time.

Figure 4 shows the unsteady pressure distributions at different radial locations in the axial position, $X_{3}=0.186$. It is shown that the unsteady pressure distributions have similar trends with the unsteady pressure distributions at $X_{2}=0.074$ for the same reason as discussed previously. For $X_{2}=0.074$, the pressure 


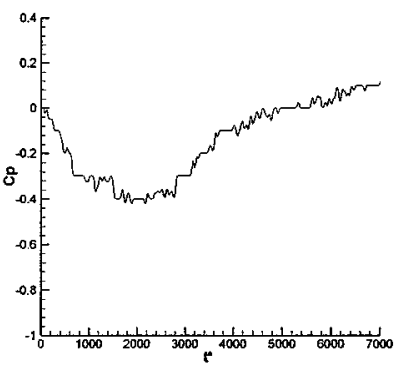

(a). $R_{1}=0.25$

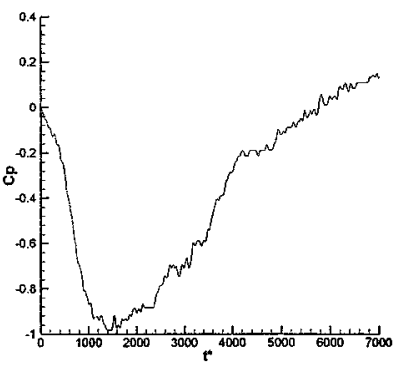

(d) $\mathrm{R}_{4}=0.67$

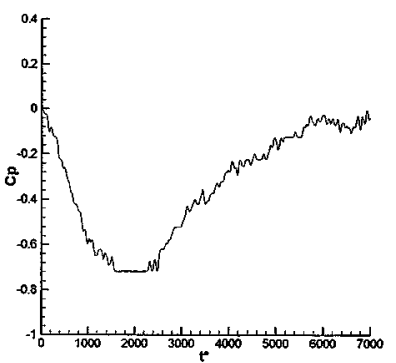

(b) $\mathrm{R}_{2}=0.33$

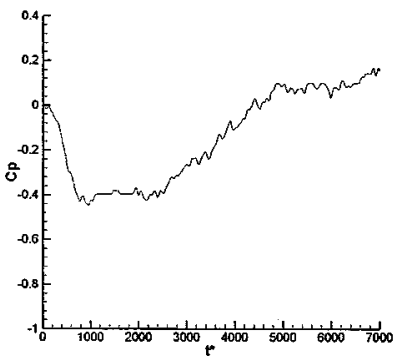

(e) $\mathrm{R}_{s}=0.83$

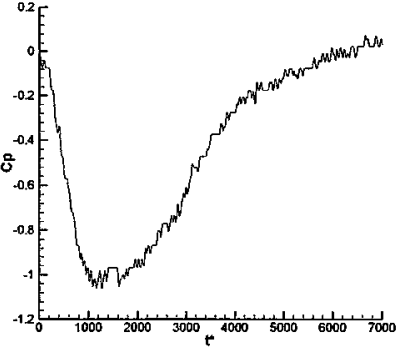

(c) $\mathrm{R}_{3}=0.50$

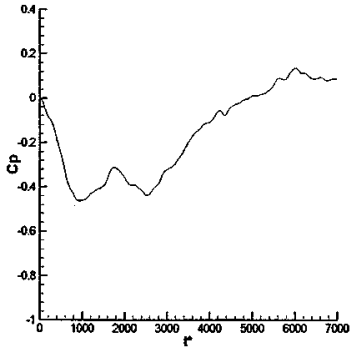

(f) $\mathrm{R}_{6}=0.92$

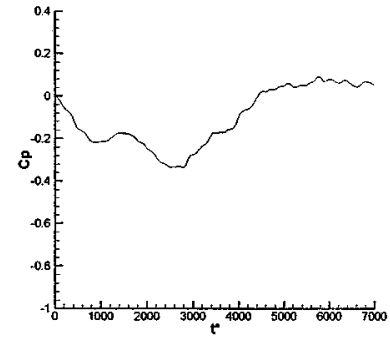

(g) $\mathrm{R}_{7}=1.00$

FIGURE 4

Unsteady pressure during the fan start at $X_{3}=0.186$.

does not change remarkably near the hub and tip of the blades. It is also shown that there exists a sharp drop of the pressure in the middle of the radius range (see Figures $4(c)$ and $4(d)$ ). This drop is more severe than those near the tip and hub. In this study, it is found that the long term steady pressure distribution along the axial location does not purely decreases or increases with the distance along the blade. It is shown in Figures 3 and 4 that the steady pressure distributions along the axial direction have different characteristics along the radial locations. Also observed in Figures 3 and 4 are the long term steady pressure increases near the hub and tip and decreases near range of the center of the blade. This observation is different from the recently steady pressure measurements of an axial fan in a wind tunnel by Mutama and Hall (1996) where the authors concluded that for $R<0.8$, the steady pressures decrease as one moves away from the fan. The present results can be explained due to the air being unable to expand freely near the hub and casing due to the wall friction. Near the hub, the air moves very slowly due to the fan design. This slow moving air near the hub restricts the expansion of the air near the hub. Therefore, the pressure variation results from the mixing process of the primary and secondary flow streams.

The total pressure measurements in the outlet of the fan have similar characteristics in different axial locations. The total pressure variations with time at $X_{3}=0.186$ are shown in Figure 5 . During the fan start phase, the air starts to move and produces a large circulation and turbulence. This strong circulation and turbulence causes a large total pressure loss. As time increases, the flow becomes relatively stable and the total pressure gradually recovers.
The unsteady pressure measurements in the fan inlet and outlet show that the flow field is strongly three-dimensional and complex. The two-dimensional analysis can only explain part of the pressure variations. The unsteady wakes are actually velocity deficiencies caused by the blockage of the mainstream flow by the rotating blades. These velocity deficiencies induce the unsteadiness of the pressure field. The casing wall and the hub have a strong influence on the development of the flow.

\section{Unsteady Pressure Field When the Fan Runs at a Constant Rotational Speed}

The theoretical analysis section above shows that the vortex formations from the trailing edge of the fan sections are a sinusoidal oscillation even though the fan rotates at a constant speed. Although the two-dimensional vortex shedding and tangential velocity dominate the outlet pressure changes with time, the three-dimensional sources, such as a centrifugal effect and flow-blade interaction, also influence the pressure field. Therefore, two-dimensional theory formulas may not explain the measurement results. The variations in the unsteady pressure distributions with time in the outlet plane are similar to all different axial positions. The pressure fluctuations with time at $X_{3}=0.184$ when the fan rotates at an operational constant speed of $1770 \mathrm{rpm}$ is shown in Figure 6. The pressure distributions show that in general the pressure fluctuation has large magnitudes near the blade tip compared with those near the middle of the blade. Figure $6(b)$ shows that the magnitudes of the pressure fluctuation at $R_{2}=0.33$ are larger than at $R_{1}=0.25$ and $R_{3}=0.50$, because the location of $R_{2}=0.33$ coincides with 


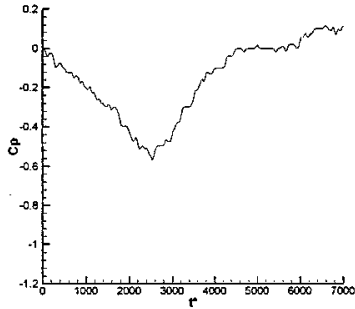

(a) $\mathrm{R}_{1}=0.25$

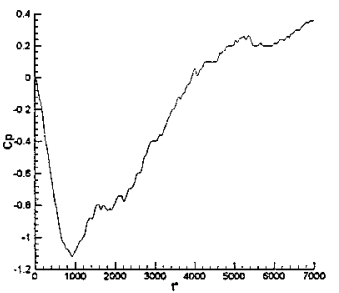

(d) $\mathrm{R}_{4}=0.67$

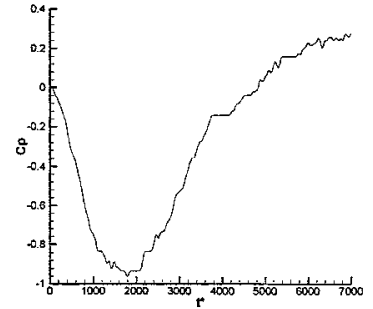

(b) $\mathrm{R}_{2}=0.33$

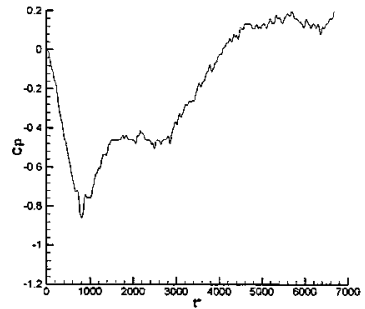

(e) $\mathrm{R}_{5}=0.83$

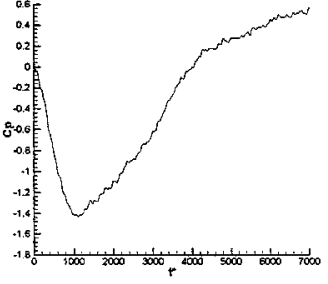

(c). $\mathrm{R}_{3}=0.50$

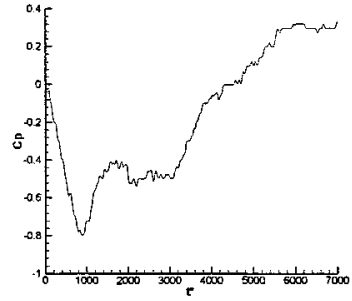

(f) $\mathrm{R}_{6}=0.92$

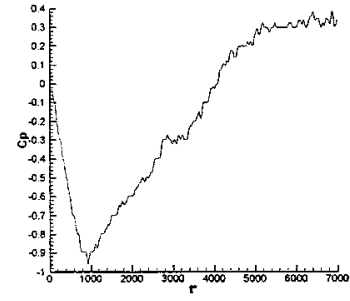

(g) $\mathrm{R}_{7}=1.00$

FIGURE 5

Unsteady total pressure during the fan start at $X_{3}=0.186$.

the location of the root of the blades. At this location, the shed vortices have strong interactions with the lower speed of the air inside the fan unit and thus produce a larger fluctuation of the velocity and pressure. Figure 6 shows that the average frequency of the pressure fluctuation is about 0.8 , which is slightly less than the fan blade rotational frequency. This observation suggests that the mechanical vibration in the fan design should be taken into account in the aerodynamic frequency rather than fan rotational frequency.
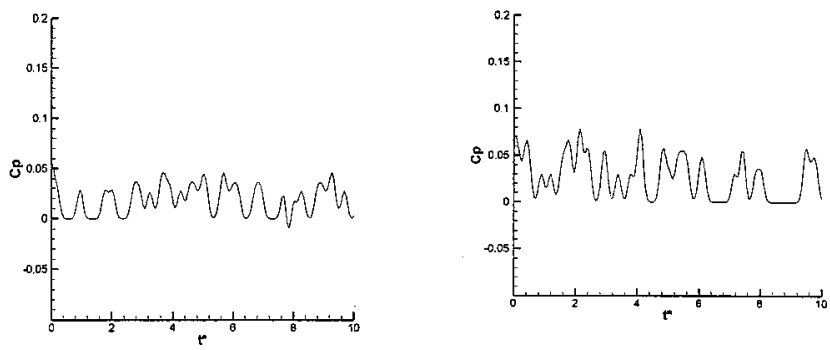

(b). $\mathrm{R}_{2}=0.33$

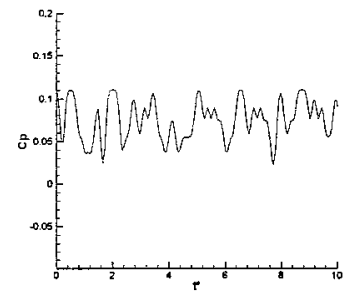

(d). $\mathrm{R}_{4}=0.67$

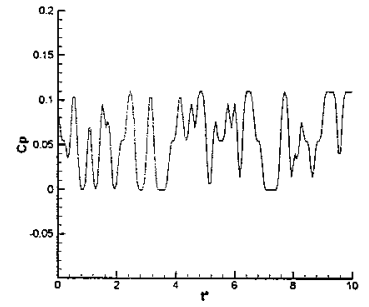

(e). $\mathrm{R}_{5}=0.83$

The unsteady pressure patterns are identical for almost all the measurement locations during the test. Long time unsteady pressure distribution at $R_{4}=0.67$ and $X_{3}=0.186$ is shown in Figure 7. This figure shows that the pressure has two types of fluctuations. One is high frequency, which is related to the fan rotation as shown in Figure 6, and the other is lower frequency, which may be related to the aerodynamic delay. It is noteworthy to point out that the unsteady pressure has a periodic characteristic, except the periodic frequency of the instantaneous velocity

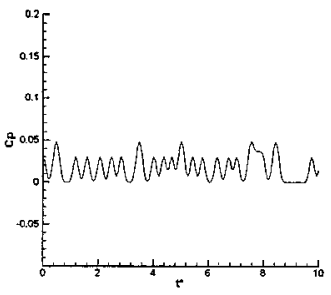

(c) $\mathrm{R}_{3}=0.50$

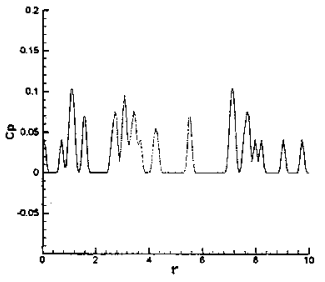

(f) $\mathrm{R}_{\mathrm{s}}=0.92$

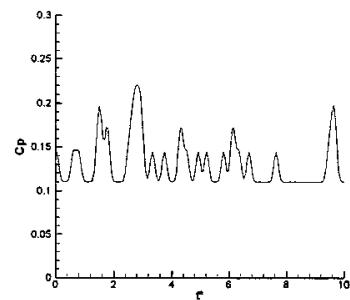

(g). $\mathrm{R}_{7}=1.00$

FIGURE 6

Unsteady pressure distributions at $X_{3}=0.186$. 


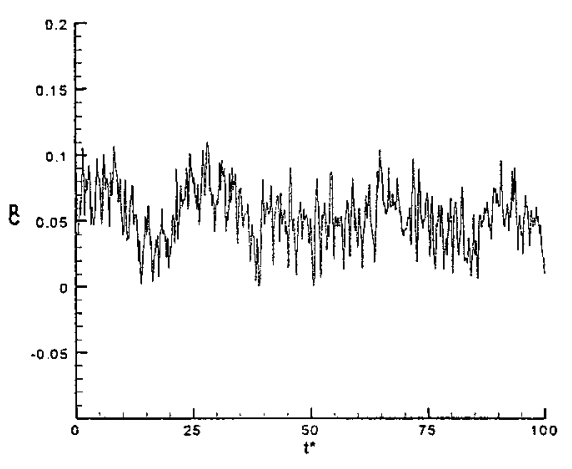

FIGURE 7

Unsteady pressure distribution at $R_{4}=0.67, X_{3}=0.074$.

profiles of the gas turbine blades as reported by Du, Han, and Ekkad (1998). It is shown that the pressure and velocity fluctuations are caused by the upstream passing wakes, for which again the testing results are in agreement with the theoretical analysis section. The early theory by Abramovich (1963) showed that by maintaining a constant rotational speed the aerodynamic behavior would be quasi-steady with minor effects resulting from the cylindrical tower wake. This study shows that this is not entirely correct. The pressure distribution demonstrated high unsteadiness even when the fan is running at a constant rotational speed.

\section{CONCLUSIONS}

The pressure distribution of the fan inlet and outlet are very important in understanding the fan performance and to investigate the reasons of any possible fan failures during the fan operation. In this study, a detailed experimental testing and a theoretical analysis of the inlet and outlet pressure distribution of the fan were performed during the fan start up and while the fan was running at a constant rotational speed. The pressure field was found to be affected significantly by the rotation characteristics of the fan and the fan unit structure. The study also showed that the pressure distribution had a strong fluctuation both at inlet and outlet of the fan during the fan start-up. This fluctuation has a strong influence on the fan structure. It is found that when the fan runs at the design condition, the fluctuation frequency of the pressure distribution is slightly lower than the fan rotational frequency.

This research effort has resulted in the systematic analysis of the selected data sets from inlet and outlet dynamic pressure fields. This study provides information on the fan unsteady aerodynamics, which helps with the fan design, performance analysis, and fan failure analysis. The experimental results demonstrated the strong three-dimensional aerodynamics associated with the fan during the fan start-up and at the design working condition. The theoretical study supported the experimental observations that the pressure oscillations depend on the fan rotational speed as well as the radius location of the fan, which is consistent with the measurement results. The experimental study presented here provides a wealth of data that can be used to identify problems during the design and can be used as CFD validation data. This database may assist in developing future design improvements. Future studies that address the dynamic pressure variation on the fan blades should be conducted.

\section{NOMENCLATURE}

$\begin{array}{ll}\mathrm{C} & \text { chord of the fan blade } \\ C_{p} & \text { pressure coefficient } \\ d & \text { diameter of fan } \\ \mathrm{g} & \text { acceleration of gravity } \\ H & \text { total head } \\ l & \text { length of the casing } \\ r & \text { fan radius } \\ R & \text { non-dimensional radius }=r / r_{\text {tip }} \\ n & \text { harmonic indices } \\ p & \text { pressure } \\ s & \text { coordinate along the streamline } \\ t & \text { time } \\ t^{*} & \text { non-dimensional time, }=t / T \\ T & \text { time of the rotation in one circle } \\ u & \text { average velocity } \\ V & \text { velocity } \\ x & \text { axial distance from the rotational plane to test point } \\ X & \text { non-dimensional axial distance }=x / r_{t i p} \\ Z & \text { Number of blades }\end{array}$

\section{Greek}

$\gamma \quad$ vortex strength

$\theta \quad$ stationary coordinate

$\omega \quad$ shaft angular speed

\section{Subscripts}

$1,2,3$, position indices

$4,5,6,7$

$b \quad$ blade or bounded vortex

$m$ reference point

$i \quad$ inlet plan

$o \quad$ outlet plan

$q \quad$ indices

$s \quad$ steady state

$s 1, s 2$ leading and trailing edge

$t \quad$ blade tip position

$w \quad$ wake

$\infty \quad$ infinite field or stagnation point

\section{REFERENCES}

Abramovich, G. N. 1963. The theory of turbulent jets. Cambridge, MA: MIT Press.

Capace, N. R., and Fleeter, S. 1989. Experimental investigation of multistage interaction gust aerodynamics. ASME Journal of Engineering for Power. 111:409-417. 
Dring, R. P., Joslyn, H. D., Hardin, L. W., and Wagner, J. H. 1982. Turbine rotor-stator interaction. ASME Journal of Engineering for Power 104:729-742.

Du, H., Han, J. C., and Ekkad, S. V. 1998. Effect of unsteady wake on detailed heat transfer coefficient and film effectiveness distributions for a gas turbine blade. ASME Journal Turbomachinery 120:808817.

Gallus, H. E., Lambertz, J., and Wallmann, T. 1980. Blade-row interaction in an axial-flow subsonic compressor stage. ASME Journal of Engineering for Power 102:169-177.

Huyer, S. A., Simms, D., and Robinson, M. C. 1996. Unsteady aerodynamics associated with a horizontal-axis wind turbine. AIAA Journal 34:1410-1419.

Kaupert, K. A., and Staubli, T. 1999. The unsteady pressure field in a high specific speed centrifugal pump impeller-Part 1: Influence of the volute. ASME Journal of Fluids Engineering 121:621626.
Kaupert, K. A., and Staubli, T. 1999. The unsteady pressure field in a high specific speed centrifugal pump impeller-Part 2: Transient hysteresis in the characteristic. ASME Journal of Fluids Engineering 121:667-632.

Minniti, R. J., Sullivan, C. J., and Mueller, T. J. 1998. A thin film sensor for the measurement of unsteady pressure. The Aeronautical Journal 102:393-398.

Mutama, K. R., and Hall, A. E. 1996. The experimental investigation of jet fan aerodynamics using wind tunnel modeling. ASME Journal of Fluids Engineering 118:322-327.

Xu, C., and Amano, R. S. 1999. The nonsymmetrical uncertainty intervals in fluid measurement system. Mechanical Engineering Research Report. Milwaukee: University of WisconsinMilwaukee.

Xu, C., and Yeung, W. W. H. 1998: Numerical study of unsteady flow around airfoil with spoiler. ASME Journal of Applied Mechanics 65:164-170. 

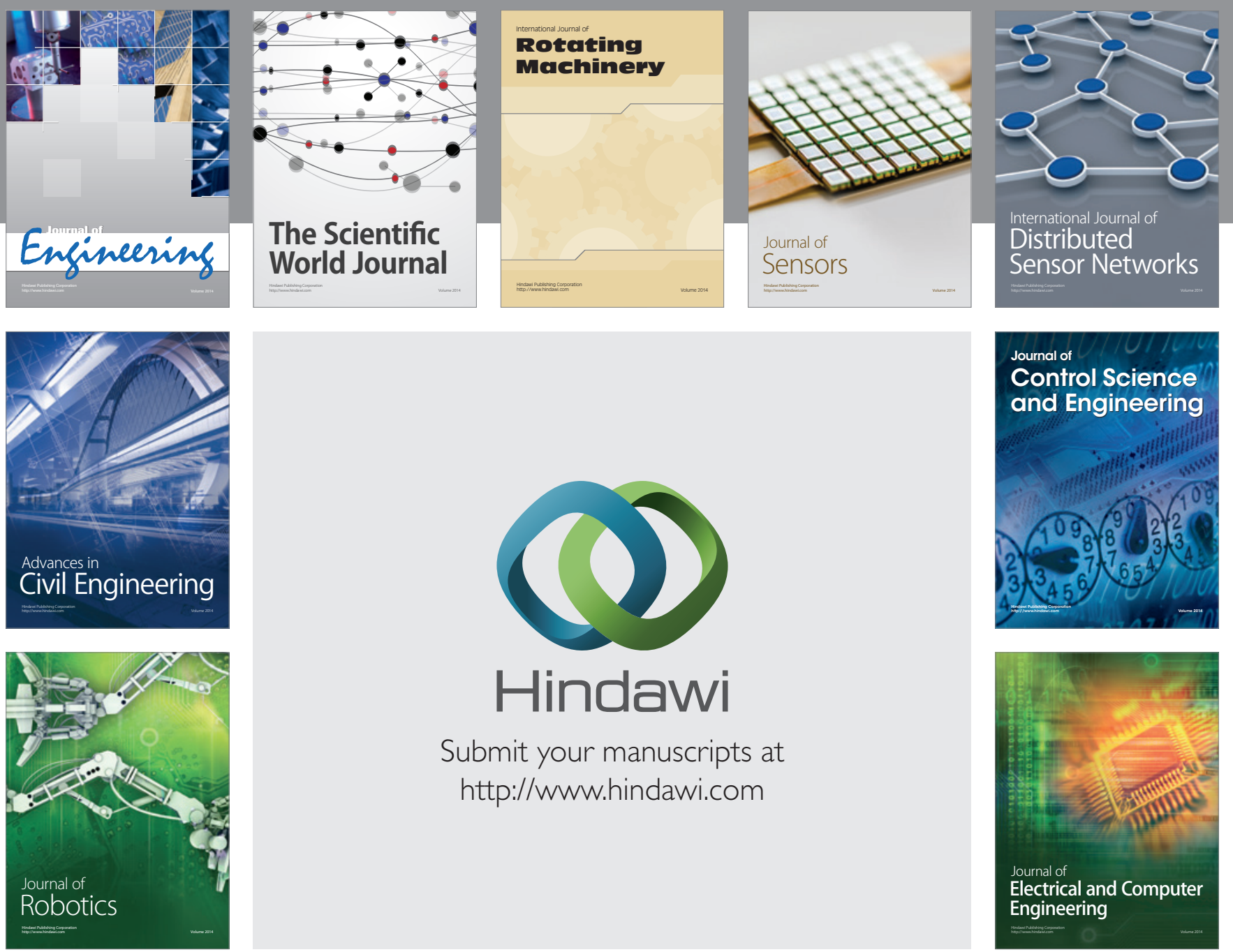

Submit your manuscripts at

http://www.hindawi.com
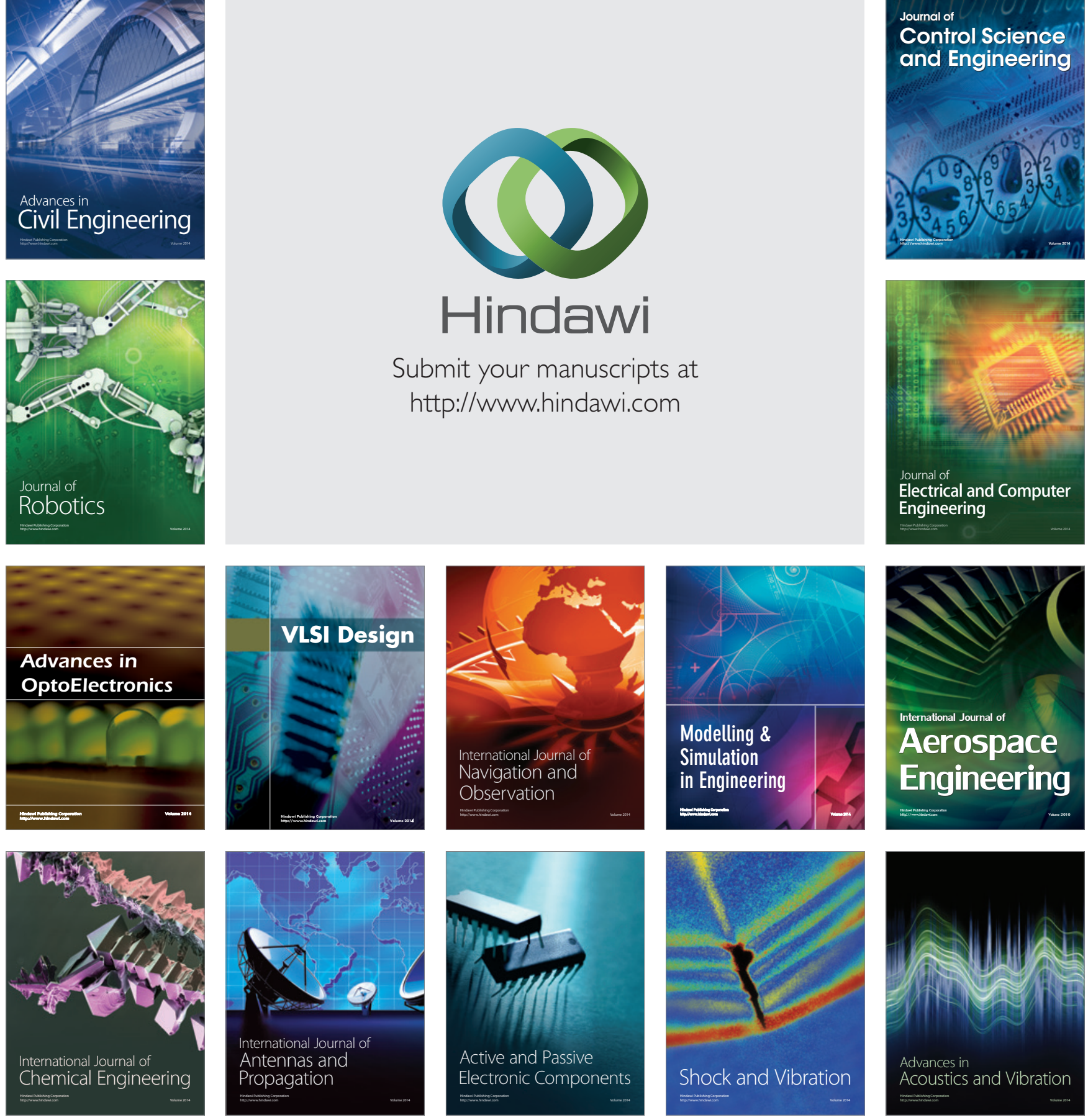